\title{
Directed Motion for Delta-Kicked Atoms with Broken Symmetries: Comparison between Theory and Experiment
}

\author{
P. H. Jones, M. Goonasekera, D. R. Meacher, T. Jonckheere, ${ }^{*}$ and T. S. Monteiro \\ Department of Physics and Astronomy, University College London, Gower Street, London WC1E 6BT, United Kingdom
}

(Received 4 August 2006; published 14 February 2007)

\begin{abstract}
We report an experimental investigation of momentum diffusion in the $\delta$-function kicked rotor where time symmetry is broken by a two-period kicking cycle and spatial symmetry by an alternating linear potential. We exploit this, and a technique involving a moving optical potential, to create an asymmetry in the momentum diffusion that is due to the classical chaotic diffusion. This represents a realization of a type of Hamiltonian quantum ratchet.
\end{abstract}

DOI: $10.1103 /$ PhysRevLett.98.073002

PACS numbers: $32.80 . P j, 05.45 . \mathrm{Mt}$

The ratchet effect, in other words the rectification of fluctuations in a system without net bias, was first proposed by Feynmann and has since formed the subject of numerous studies [1,2]. Recently, there has been further interest and investigations of Hamiltonian chaotic ratchets, where the extrinsic noise is replaced by deterministic chaos. Hamiltonian systems offer the additional possibility of a fully quantum ratchet, where some form of directed transport appears in the context of coherent wave dynamics; other types of ratchets in dissipative and noisy quantum systems, corresponding to coherence times which are relatively short, have also been proposed [3]. Previous studies of chaotic Hamiltonian ratchets [4] indicated that directed motion arises if certain symmetries are broken, but persists only in the presence of mixed phase-space dynamics (e.g., a bounded classical phase space with a mixture of regular tori and chaotic regions). The quantitative analysis of the directed current then relies on the details of the classical phase space [2].

In $[5,6]$ an alternative theoretical proposal for chaotic but asymmetric momentum diffusion, aimed at a realization with cold atoms in far-detuned pulsed optical lattices, was presented. Experiments with cold atoms in neardetuned, driven optical lattices had already been shown to provide realizations of classical Brownian and dissipative ratchets [7]. Far-detuned lattices minimize decoherence effects; hence, they provided the clearest demonstrations of Hamiltonian quantum chaotic dynamics [8]. In particular, cold atoms in $\delta$-kicked optical lattices can realize the dynamics of the chaotic quantum kicked rotor and show the effect of dynamical localization (DL): the momentum diffusion of the cold atoms follows approximately the classical chaotic rate, $\left\langle p^{2}\right\rangle \approx D t$, up to a time scale $t^{*} \sim D / \hbar^{-2}$, after which the diffusion stops and the quantum momentum probability distribution $N(p)$ localizes, with a variance $\left\langle p^{2}\right\rangle^{1 / 2}=L \sim D / \hbar$. DL is a quantum coherent effect due to destructive wave interference [9].

The directed chaotic transport mechanism of $[5,6]$ is generic in character: a quantum kicked rotor with broken time and space symmetry diffuses asymmetrically for a finite time scale $t_{R}$. An ensemble of classical particles initially with $\langle p(t=0)\rangle=0$ will end up with nonzero current $\left\langle p\left(t=t_{R}\right)\right\rangle \neq 0$. However, in the classical case, the kinetic energy and hence the momentum width $L \sim$ $\sqrt{\left\langle p^{2}\right\rangle}=\sqrt{D t}$ grow without limit as $t \rightarrow \infty$. For a ratchet in an unbounded phase space (such as a chaotic system), it is thus most useful to consider the behavior of the relative asymmetry $I_{R}(t)=\langle p(t)\rangle / L$. Hence, for the classical dynamics, $I_{R}(t \rightarrow \infty) \rightarrow 0$. In contrast, for the quantum dynamics, a new and modified form of localization, asymmetric dynamical localization (ADL), was found in $[5,6]$ to arrest the diffusion and "freeze" the asymmetry. Hence the relative asymmetry $I_{R}(t \rightarrow \infty) \rightarrow\left\langle p\left(t_{R}\right)\right\rangle /$ $\sqrt{D t^{*}}$; i.e., it tends to a constant value. This may be considered a type of quantum ratchet. Other theoretical proposals for Hamiltonian regular or chaotic directed motion have subsequently also been published by several groups [10].

In this Letter we report experimental realizations of the chaotic kicked rotor with the broken time and space symmetry proposed in [5,6], which permit quantitative comparisons with the analytical results for the "ratchet time" $t_{R}$ and the periodic "current reversals" expected from the theoretical model. We present an accurate formula for the classical ratchet current (a simplified formula was presented in [11]). Good agreement is obtained between theory and experiment. To our knowledge, this experiment remains the only realization of directed transport in a Hamiltonian quantum system.

An optical lattice formed by two counterpropagating laser beams may be used to trap laser-cooled atoms in a one-dimensional periodic potential. Here, an accelerating optical lattice was sometimes also used to apply an additional "rocking" linear potential. In a frame with acceleration $a$, the Hamiltonian has an additional inertial term [12]:

$$
H=\frac{p^{2}}{2 M}+V_{0} \cos \left(2 k_{L} x\right) \pm \operatorname{Max},
$$

where $M$ is the mass of the atom, $k_{L}=2 \pi / \lambda$ the laser 
wave vector, and $V_{0}$ the potential depth. If the optical lattice is applied as a series of short ( $\delta$-function) pulses with period $T$, then we may, as for the usual $\delta$-kicked rotor, write the Hamiltonian including the rocking potential in dimensionless form:

$$
\mathcal{H}=\frac{\rho^{2}}{2}+\sum_{n}\left[K \cos (\phi)+A(-1)^{n} \phi\right] \delta(\tau-n),
$$

where $K$ is the stochasticity parameter which describes the strength of the kick. Here $\rho=2 T k_{L} p / M$ is a scaled momentum, $\phi=2 k_{L} x$ a scaled position, $\tau=t / T$ a scaled time, and $\mathcal{H}=8 \omega_{R} T^{2} H / \hbar$ the scaled Hamiltonian, and $A=2 k_{L} a T^{2}$ is the effective strength of the linear potential. The commutation relation $[\phi, \rho]=i 8 \omega_{R} T$ gives the scaled unit of system action or effective Planck constant $\hbar_{\text {eff }}=8 \omega_{R} T\left(\omega_{R}\right.$ the atomic recoil frequency) which may be controlled through the period of the pulses.

For a $\delta$-kicked rotor, to lowest order, the momentum diffusion rate is the uncorrelated "random walk" rate $D_{0} \simeq$ $K^{2} / 2$. Corrections to the random walk rate arise from short-range correlations between kicks, reflecting a shortterm "memory" in the system [13]. The leading nonzero correction is $C(2)=K^{2} J_{2}(K)$, a 2-kick correlation (simply put, it implies a memory effect between kick $n$ and kick $n+2)$. It persists deep into the chaotic limit since it decays slowly: $C(2) / D_{0} \sim K^{-1 / 2}$, so remains important even if, say, $K \approx 50$. In [6] it was shown that for the classical $\delta$-kicked rotor (with $A \neq 0$ ) where time symmetry is broken by a kicking cycle of periods $T(1+b): T(1-b)$ (where $b \ll 1$ ), then, for short times, this 2-kick correlation takes a novel, quite different form. The net effect is that even pure chaotic diffusion can produce an asymmetry in the momentum distribution $N(\rho)$.

We consider now an ensemble of particles with initial $(t=0)$ momentum distribution strongly peaked about a value $\rho_{L}$, in the (nonaccelerated) lattice frame, i.e., $N_{0}(\rho) \approx \delta\left(\rho-\rho_{L}\right)$. Then, here we extend the work in [6]: using the method of [13], to obtain an explicit formula for the asymmetry $I(t)=\left\langle\left(\rho-\rho_{L}\right)\right\rangle$ at arbitrary times:

$$
I(t)=I_{0} \sin \left[(1-b) A-2 b \rho_{L}\right] F(t),
$$

where the maximum current is $I_{0}=\frac{-K J_{1}(2 K b)}{1-J_{0}(2 K b)^{2}} \times$ $\left\{J_{0}(2 K b) J_{2}[(1-b) K]+J_{2}[(1+b) K]\right\}$ and the time dependence is given by $F$ where $1 \geq F(t) \geq 0$ :

$$
F(t)=1-J_{0}(2 K b)^{2 t-2} .
$$

For $t$ small, $F(t) \sim t$ grows linearly with time, but for $t \gg$ $t_{R} \approx 1 /(K b)^{2}$, it saturates, i.e., $F(t) \rightarrow 1$. Hence $t_{R}$ is the classical time scale for the current to develop; if dynamical localization occurs too quickly, i.e., $t * \ll t_{R}$, no appreciable quantum effect is observed. Conversely, if $t^{*} \gg$ $t_{R}$, the asymmetry is negligible compared with $L$ [the asymptotic variance of $N(\rho)]$. Optimally, we require $t^{*} \sim t_{R}$.
For a ratchet effect, as usually understood, a distribution initially with zero average momentum $\langle\rho\rangle=0$ and $\rho_{L}=$ 0 , i.e., in the rest frame of a potential (giving no net bias), evolves to an asymptotic distribution with $\langle\rho\rangle \geq 0$. In the present experiment, this requires $A \neq 0$ : for example, for $A=\pi / 2$ and $\rho=0$, if at $t=0,\langle\rho\rangle=0$, we obtain $I\left(t>t^{*}\right) \simeq I_{0}$. However, in order to fully investigate the underlying mechanism, here we also investigated extensively starting conditions with nonzero initial momentum, i.e., $\rho \neq 0$.

In our experiment we use laser-cooled cesium atoms in a far-off resonant pulsed optical lattice. The lattice is formed by two horizontal counterpropagating laser beams, $1 / e$ radius $(0.95 \pm 0.05 \mathrm{~mm})$, with parallel linear polarizations which produces a spatial variation of the ac Stark shift proportional to the local intensity, and which hence is sinusoidal. Further details of the setup are found in [14].

To investigate starting conditions with nonzero mean momentum we have used a moving optical lattice formed by laser beams with a controlled frequency difference to make the kicking potential, so that atoms which are stationary in the laboratory frame have a momentum $\rho_{L}$ in the rest frame of the optical potential. This is achieved by driving two acousto-optic modulators at frequencies that differ by $2 \Delta f$, such that the atomic momentum in the rest frame of lattice is $\rho_{L}=m \lambda^{2} \Delta f \hbar_{\text {eff }} / 4 \pi \hbar$. Using this technique, $\rho_{L}$ may be varied over a large range in order to sample several periods of the oscillation of the asymmetric diffusion without the beams becoming significantly misaligned from the cloud of cold atoms.

For these experiments the period of the kicks is $T=$ $9.47 \mu \mathrm{s}$ and pulses are square with duration typically $t_{p}=$ $296 \mathrm{~ns}\left(t_{p} / T=1 / 32 \leq b\right)$, which is sufficient for there to be no substantial effects on the diffusion constant due to the finite temporal width of the kicks in the region of $\rho_{L} \approx$ 0 [15] (for larger $\rho_{L}$, these effects become important and start to affect the data). An investigation of the effects on the momentum diffusion arising the finite width of the kicks was presented in [14]. We were able to investigate values of the parameters $K=2 \rightarrow 5$ (with order 10\% error arising mainly from the measurement of the beam intensity), $h_{\text {eff }}=1$, and values of $b=1 / 8 \rightarrow 1 / 32$. Values of $K$ close to the first maximum of the Bessel function $J_{2}(K)$ may be expected to produce the largest maximal currents $I_{0}$ and hence the clearest experimental signature. For values of $K \approx 2-5$ there are still stable islands in the classical phase space; nevertheless, these are sufficiently small: for $b \neq 0$, good quantitative agreement with calculated chaotic diffusive rates is obtained for lower $K$ than for the standard map [6].

Finally, for experiments with $A \neq 0$, the linear rocking term of alternating sign was included by accelerating the optical lattice [16]. This is done by modulating the frequency of one of the laser beams in a linear manner by an amount $\pm \delta f$ in the time of the kick period $T$. The dimensionless potential gradient $A$ is related to the magnitude 


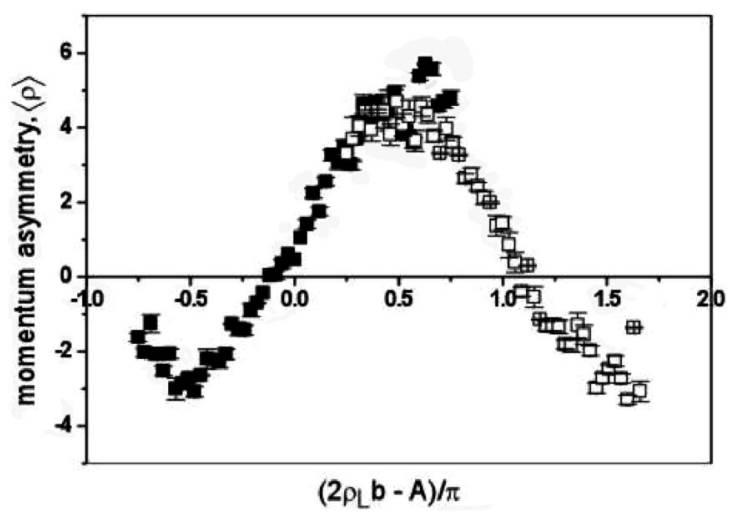

FIG. 1. Demonstration of a type of quantum "ratchet effect." The graph shows the final momentum asymmetry $\langle\rho\rangle$ vs $\Phi=$ $\left(2 \rho_{L} b-A\right) / \pi$ for $K=2.6, b=1 / 16$, and $\hbar_{\text {eff }}=1$. Solid squares are data for $\rho_{L}=0$, open squares $\rho_{L}=8 \pi$. For the maximum at $\Phi=0.5$, the cloud is initially at rest relative to the optical lattice (i.e., $\rho_{L}=0$ ) and has no asymmetry; after 120 kicks, there is a constant "ratchet current" $\langle\rho\rangle \approx 4$.

of the frequency modulation (acceleration of the lattice) by $A=2 \pi t_{p} \delta f$ for finite square pulses of width $t_{p}$. Accelerating the potential thus provides a simple way of controlling the magnitude of $A$ and hence controlling the phase shift of the momentum-dependent diffusion constant in order to make it locally asymmetric around zero momentum. As the maximum frequency modulation amplitude allowed by the radio-frequency synthesizers in our apparatus was $\pm 1.25 \mathrm{MHz}$, this limits the range of $A$ achievable to $\pm 3 \pi / 4$. In order to observe one complete oscillation of the momentum diffusion constant, for some experiments an additional constant frequency offset was introduced between the laser beams such that in the rest frame of the lattice the mean atomic momentum was $\rho_{L}=$ $8 \pi$.

Figure 1 shows the asymmetry $\langle\rho\rangle$ (combining $\rho_{L}=0$ and $\left.\rho_{L}=8 \pi\right)$ plotted as a function of $\Phi=\left(2 \rho_{L} b-\right.$ $A) / \pi \approx\left[2 \rho_{L} b-A(1-b)\right] / \pi$ suggested by Eq. (3). In particular, the data at $\Phi=0.5$, corresponding to $\rho_{L}=0$, hence represent a ratchet current $I_{0} \approx 4$ obtained in the rest frame of the potential. The behavior is in good agreement with quantum simulations carried out here, which yielded $I\left(t \geq t^{*}\right) \simeq I_{0} \sin \Phi \simeq 3.8 \sin \Phi$. The corresponding classical formula from Eq. (3) yields a much larger current $I_{0} \approx 7.5$. For these values of $K$ and $b$, the result is close to the simplified classical current formula $I(t \rightarrow \infty) \approx$ $K^{2} \frac{J_{2}(K)}{b} \sin \Phi$ obtained in [11].

In Fig. 2 the dependence on $\langle\rho\rangle$ on $b$ is tested more extensively for $A=0$ and is seen to oscillate with a period $\pi / b$ and is consistent with $I_{0} \propto 1 / b$, in agreement with theory. However, in order to test experimentally the time dependence of the classical correlations [i.e., the behavior of $F(t)$ above], further experiments for smaller $\hbar \approx 1 / 4$, for which there is closer agreement for classical and quantal diffusion (i.e., $t^{*} \approx t_{R}$ ), were obtained. Hence in Fig. 3 the growth in time of the momentum asymmetry $\langle\rho\rangle$ is

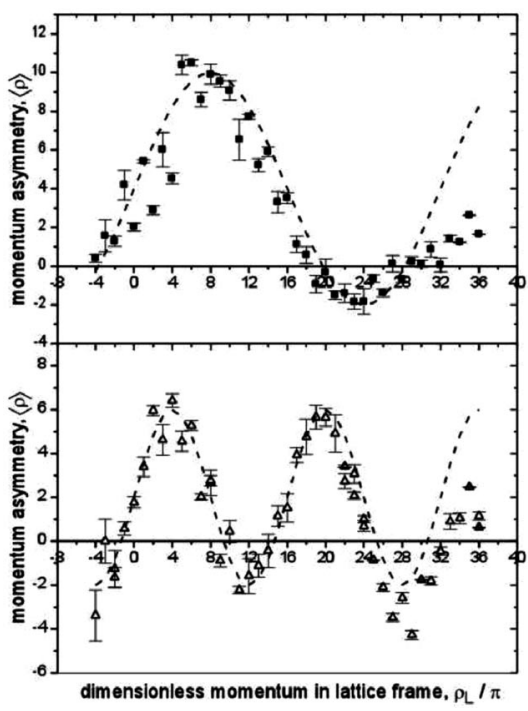

FIG. 2. Behavior as a function of $b$ and initial momentum $\rho_{L}$. Momentum asymmetry vs starting momentum $\rho_{L}$ in the lattice frame for $K=3.3, \hbar_{\mathrm{eff}}=1$, and $b=1 / 32$ (solid squares) and $b=1 / 16$ (open triangles). The asymmetry is consistent with the theoretical prediction of Eq. (3), i.e., $\langle\rho\rangle \propto I_{0} \sin 2 \rho_{L} b$ and (approximately) $I_{0} \propto 1 / b$.

shown for the quantal, classical [i.e., Eq. (3)], and experimental values and for $K=2.1, \hbar=1 / 4$, and $A=0$. The agreement between all three is quite good. Strikingly, there is, for these parameters, near-perfect agreement between the quantum calculation and the classical formula since $t^{*} \sim \pi^{2} /(K b)^{2} \sim t_{R}$. The agreement with experiment is reasonable too, but the experimental curves experience slight distortion due to the momentum boundary due to the finite duration of the pulses [14]. While the relatively small value of $\hbar=1 / 4$ considered yields excellent classical-quantal agreement, it means that the momentum

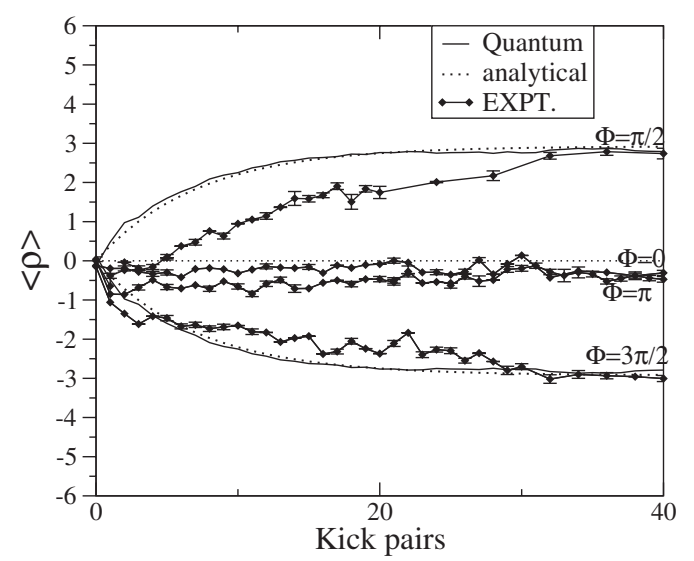

FIG. 3. Time dependence of the momentum asymmetry for $K=2.1, \hbar_{\mathrm{eff}}=1 / 4$, and $b=1 / 8$ (solid squares), for experimental, classical, and quantal results in a regime where $t^{*} \simeq t_{R}$. Good agreement for all three is shown. The results confirm the validity of the time dependence of Eq. (3). 


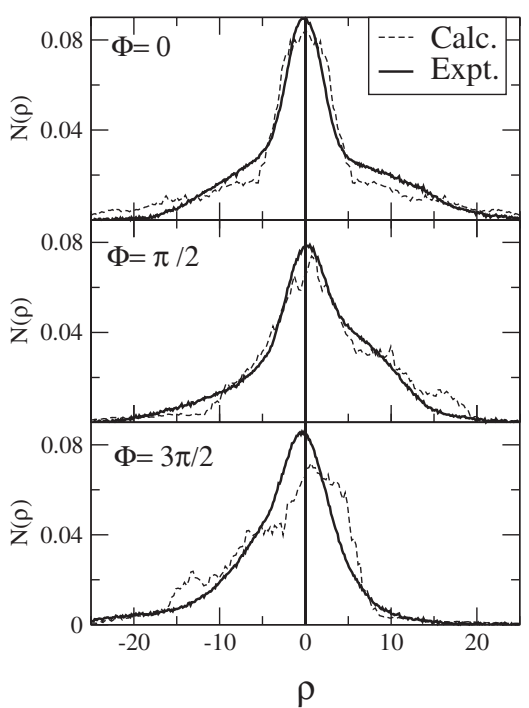

FIG. 4. Experimental and quantal momentum distributions after 240 kicks, showing that quantum coherence is sufficiently maintained that asymmetric dynamical localization persists for long times. This shows that the quantum ratchet effect persists asymptotically. Both (b) and (c) show a large relative asymmetry $\langle p\rangle / L$. The corresponding classical momentum is not shown: while $\langle p\rangle$ is similar for the quantum and classical results, the width (hence the kinetic energy) of the classical distribution grows without limit, i.e., $L \sim \sqrt{D t} \rightarrow \infty$. The relative asymmetry $\langle p\rangle / L \rightarrow 0$ asymptotically and the asymmetry becomes less apparent at long times.

boundary is rather closer in, at $\rho \simeq 16 \pi$ (the theoretical values, with perfect $\delta$ kicks, are obviously unaffected). Figure 4 shows the long time behavior but otherwise the same parameters as Fig. 3. The calculated quantal and experimental momentum distributions preserved the frozen ADL distribution [in practice decoherent processes will lead to slow loss of ADL in the experimental $N(p)$ ], but Fig. 4 shows that the qualitative behavior remains after 120 kick pairs. We note that the method for evaluation of the experimental $N(\rho)$ will tend to somewhat overestimate $\langle\rho\rangle$; conversely, the theoretical results are for an initial (Gaussian) distribution of momentum width $\sigma_{p}=1$; for larger initial momentum widths (and for the experiment $\sigma_{p} \approx 4$ ), there will be a damping of the current $I_{0} \rightarrow$ $I_{0} \exp \left(-4 \sigma_{p}^{2} b^{2}\right)$. Although the near exact quantitative agreement at $t \rightarrow \infty$ is somewhat fortuitous, quantal and experiment are in quite good agreement. The width of the classical distribution, in contrast, continues to grow without limit for chaotic and unbounded dynamics, i.e., $L \sim$ $\sqrt{\left\langle p^{2}\right\rangle} \rightarrow \infty$; hence the classical relative asymmetry $\langle p\rangle / L$ tends to zero at long times.

In summary, we have demonstrated experimentally directed transport which relies on the persistence of coherent, unitary quantum evolution over the full time scale of the experiment. This represents a type of Hamiltonian quantum ratchet.
We would like to thank H. E. Saunders-Singer for useful discussions and EPSRC and UCL for financial support.

*Present address: Centre de Physique Théorique, Campus de Luminy, case 907, 13288 Marseille cedex 9, France.

[1] R. D. Astumian and P. Hanggi, Phys. Today 55, No. 11, 33 (2002).

[2] P. Reimann, Phys. Rep. 361, 57 (2002).

[3] P. Reimann, M. Grifoni, and P. Hanggi, Phys. Rev. Lett. 79, 10 (1997).

[4] T. Dittrich, R. Ketzmerick, M.-F. Otto, and H. Schanz, Ann. Phys. (Berlin) 9, 755 (2000); H. Schanz, M.-F. Otto, R. Ketzmerick, and T. Dittrich, Phys. Rev. Lett. 87, 070601 (2001); S. Flach, O. Yevtushenko, and Y. Zolotaryuk, Phys. Rev. Lett. 84, 2358 (2000); T. Cheon, P. Exner, and P. Seba, J. Phys. Soc. Jpn. 72, 1087 (2003).

[5] T. S. Monteiro, P. A. Dando, N. A. C. Hutchings, and M. R. Isherwood, Phys. Rev. Lett. 89, 194102 (2002); N. A. C. Hutchings, M. R. Isherwood, T. Jonckheere, and T. S. Monteiro, Phys. Rev. E 70, 036205 (2004).

[6] T. Jonckheere, M. R. Isherwood, and T. S. Monteiro, Phys. Rev. Lett. 91, 253003 (2003).

[7] P. H. Jones, M. Goonasekera, and F. Renzoni, Phys. Rev. Lett. 93, 073904 (2004).

[8] F. L. Moore, J.C. Robinson, C.F. Barucha, Bala Sundaram, and M. G. Raizen, Phys. Rev. Lett. 75, 4598 (1995); B. G. Klappauf, W.H. Oskay, D. A. Steck, and M. G. Raizen, Phys. Rev. Lett. 81, 1203 (1998); J. Ringot, P. Szriftgiser, J. C. Garreau, and D. Delande, Phys. Rev. Lett. 85, 2741 (2000); G. Behinaein, V. Ramareddy, P. Ahmadi, and G. S. Summy, Phys. Rev. Lett. 97, 244101 (2006).

[9] G. Casati, B. V. Chirikov, F. M. Izraelev, and J. Ford, in Stochastic Behavior in Classical and Quantum Hamiltonian Systems, Lecture Notes in Physics Vol. 93 (Springer, Berlin, 1979), p. 334; S. Fishman, D. R. Grempel, and R.E. Prange, Phys. Rev. Lett. 49, 509 (1982).

[10] J. Gong and P. Brumer, Phys. Rev. E 70, 016202 (2004); E. Lundh and M. Wallin, Phys. Rev. Lett. 94, 110603 (2005); G. G. Carlo, G. Benenti, G. Casati, and D.L. Shepelyansky, Phys. Rev. Lett. 94, 164101 (2005); D. Poletti, G. G. Carlo, and Li Baowen, Phys. Rev. E 75, 011102 (2007); J. Gong and P. Brumer, Phys. Rev. Lett. 97, 240602 (2006).

[11] G. Hur, C. E. Creffield, P. H. Jones, and T. S. Monteiro, Phys. Rev. A 72, 013403 (2005).

[12] K. W. Madison, M. C. Fischer, and M. G. Raizen, Phys. Rev. A 60, R1767 (1999).

[13] A. B. Rechester and R. B. White, Phys. Rev. Lett. 44, 1586 (1980); A. J. Lichtenberg and M. A. Lieberman, Regular and Chaotic Dynamics (Springer, New York, 1992).

[14] P. H. Jones, M. Goonasekera, H. E. Saunders-Singer, and D. R. Meacher, Europhys. Lett. 67, 928 (2004).

[15] B. G. Klappauf, W. H. Oskay, D. A. Steck, and M. G. Raizen, Physica (Amsterdam) 131D, 78 (1999).

[16] I. H. Deutsch, P. M. Alsing, J. Grondalski, S. Ghose, D. L. Haycock, and Poul S. Jessen, J. Opt. B 2, 633 (2000). 